\title{
Examining Diffusion Networks and Identifying Opinion Leaders: A Case Study of the AIChE Concept Warehouse
}

\section{Dr. Debra M. Gilbuena, Oregon State University}

Debra Gilbuena is a postdoctoral scholar in the School of Chemical, Biological, and Environmental Engineering at Oregon State University. Debra has an M.BA, an M.S, and four years of industrial experience including a position in sensor development. Sensor development is also an area in which she holds a patent. She currently has research focused on student learning in virtual laboratories and the diffusion of educational interventions and practices.

\section{Christina Smith, Oregon State University}

Christina Smith is a graduate student in the School of Chemical, Biological, and Environmental Engineering at Oregon State University. She received her B.S. from the University of Utah in chemical engineering and is pursuing her $\mathrm{PhD}$ also in chemical engineering with an emphasis on engineering education. Her research interests include diffusion of innovations and student personal epistemology.

\section{Dr. Bill Jay Brooks, Oregon State University}

Bill Brooks is a postdoctoral scholar in the School of Chemical, Biological, and Environmental Engineering at Oregon State University. His Ph.D used written explanations to concept questions to investigate technology mediated active learning in the undergraduate chemical engineering classroom. He current interests involve using technology to enhance educational practices in promoting conceptual understanding. He is the primary programmer of the AIChE Concept Warehouse and his current focus is on its continued development, specifically creating and integrating Interactive Virtual Labs.

\section{Dr. Milo Koretsky, Oregon State University}

Milo Koretsky is a Professor of Chemical Engineering at Oregon State University. He received his B.S. and M.S. degrees from UC San Diego and his Ph.D. from UC Berkeley, all in Chemical Engineering. He currently has research activity in areas related engineering education and is interested in integrating technology into effective educational practices and in promoting the use of higher-level cognitive skills in engineering problem solving. His research interests particularly focus on what prevents students from being able to integrate and extend the knowledge developed in specific courses in the core curriculum to the more complex, authentic problems and projects they face as professionals. Dr. Koretsky is one of the founding members of the Center for Lifelong STEM Education Research at OSU. 


\title{
Examining Diffusion Networks and Identifying Opinion Leaders: A Case Study of the AIChE Concept Warehouse
}

\begin{abstract}
Propagation is a widespread goal for educational innovations. If an innovation is effective in one environment, developers usually desire to share it with other instructors and institutions to have a larger impact and improve education more broadly. Additionally, funding agencies like the National Science Foundation require a "broader impact" component in all grant proposals. One aspect commonly missing when an innovation is shared is a reflective, evidence-based description of the process as the innovation moves from the home institution to other institutions with different faculty, different students and a different culture. E.M. Rogers put forth a theory, Diffusion of Innovations, that offers one framework with which to examine this process. In this context, Rogers describes diffusion as "the process in which an innovation is communicated through certain channels over time among the members of a social system.”
\end{abstract}

We report on the first two years of propagation of the AIChE Concept Warehouse (CW), a database-driven website developed to support the chemical engineering education community's use of concept-based pedagogies. We focus on examining the diffusion networks and how they change over time. We identify opinion leaders who appear to have played a key role in the innovation's propagation. We also discuss instances where the tool appears to have been promoted without active involvement on the part of the developers. Our purpose is to highlight aspects of diffusion that other innovators may want to consider, provide an example of how these aspects can be examined in the early stages of an innovation's life, and learn how we can further support propagation of this innovation. We ask the following research questions: Through which diffusion networks has the Concept Warehouse been propagated? Who are the opinion leaders influencing its propagation and how do they promote this tool?

AIChE Concept Warehouse instructor applicants ( $\mathrm{N}>300)$ were asked, "How did you hear about us?” Network analysis was performed using network diagrams representing different points in time as visual representations of the network connections and how they changed. Further analysis of the most recent sociogram was used to identify instructors who referred this tool to four or more other users. These instructors are termed opinion leaders.

\section{Introduction}

Propagation is a widespread goal of education materials development. If an educational innovation is effective in one environment, many developers want to share it with other instructors and institutions to have a larger impact and improve education more broadly. Additionally, funding agencies like the National Science Foundation require a "broader impact" component in all grant proposals. One aspect commonly missing when an innovation is shared is a reflective, evidence-based description of the process as the innovation moves from the home institution to other institutions with different faculty, different students and a different culture. E.M. Rogers put forth a theory, Diffusion of Innovations, that offers one framework with which to examine this process. In this context, Rogers describes diffusion as "the process in which an innovation is communicated through certain channels over time among the members of a social system."1 
Conceptual learning is critical to developing problem solving skills in chemical engineering. Many engineering educators and industry partners emphasize the need for students to apply their knowledge to new and challenging problems. ${ }^{2}$ In order to do so, students must learn with understanding. ${ }^{3}$ A lack of conceptual understanding has been shown to severely restrict students' ability to solve new problems, since they do not have the functional understanding to use their knowledge in new situations. ${ }^{4}$ However, science and engineering classrooms often reward students more for rote learning than for conceptual understanding. ${ }^{5,6}$ There is clearly a need for more emphasis on conceptual understanding and concept-based instruction.

We report on the first two years of propagation of the AIChE Concept Warehouse (CW), a database-driven website developed to support the chemical engineering education community's use of concept-based pedagogies. The CW contains approximately 2000 concept-based clicker questions (or ConcepTests) and ten Concept Inventories for core chemical engineering classes. We focus on diffusion networks, particularly opinion leaders who have played a key role in the innovation's propagation. Our purpose is to highlight aspects of diffusion that other innovators may want to consider, provide an example of how these aspects can be examined in the early stages of an innovation's life, and learn how we can further support propagation of this innovation. We ask the following research questions:

1. Through which diffusion networks has the Concept Warehouse been propagated and how have they changed over time?

2. Who are the opinion leaders influencing its propagation and how do they promote this tool?

\section{Background \& Theoretical Framework AIChE Concept Warehouse}

\section{Overview}

The AIChE Concept Warehouse (CW) is a database-driven website developed to lower the activation barrier for faculty to use conceptual instruction and assessment so that many more chemical engineering faculty incorporate concept-based learning into their classes. Conceptbased instruction (e.g., ConcepTests, concept inventories) often depends on high quality concept questions. These questions can be time consuming and difficult to construct, posing one of the biggest barriers keeping faculty from implementing this type of pedagogy. ${ }^{7,8}$

This tool can be used throughout the core ChE curriculum (Material and Energy Balances, Thermodynamics, Transport Phenomena, Kinetics and Reactor Design, and Materials Science). Currently the AIChE Concept Warehouse has approximately 2000 concept questions (ConcepTests) and ten Concept Inventories available for searching, viewing, and using in courses through the user interfaces. Student and instructor interfaces are available for the community at http://cw.edudiv.org, and university faculty can obtain an account through this site. There are currently over 140 institutions and over 370 accounts registered with the AIChE Concept Warehouse.

A screen shot of the AIChE Concept Warehouse ConcepTest search page is shown in Figure 1. In order to maximize compatibility and minimize complexity, an effort was made to design the instructor interface to match with the current practices of new users, or potential adopters, to be familiar and user-friendly. One way of accomplishing this design objective was to predict and 
accommodate different ways users might leverage the AIChE Concept Warehouse. The next subsection presents the different predicted modes of use for potential adopters.

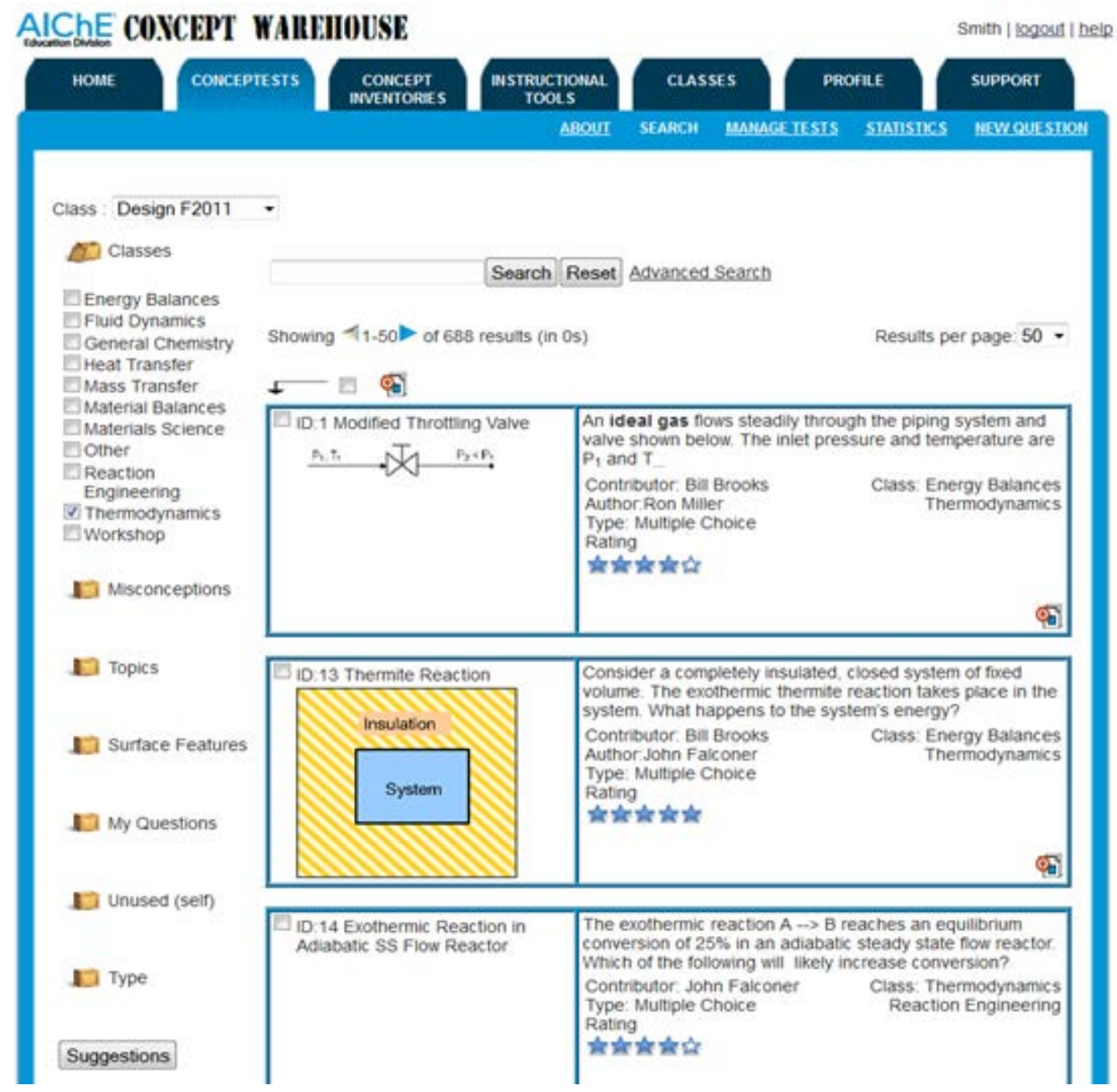

Figure 1. Screenshot of the ConcepTest search page of the AIChE Concept Warehouse

\section{Modes of Use}

There are four ways that the developers originally foresaw new users implementing the AIChE Concept Warehouse in their classes. These four modes of use are supported in the AIChE Concept Warehouse through Quick Start guides and video walkthroughs. These guides are intended for new users to facilitate their initial use. In addition, faculty may actively contribute by adding their own questions to the database.

Table 1 gives a summary of these modes of use. At all levels of use, instructors can create a class as well as find and select a set of concept questions to create a ConcepTest.

1. Offline refers to not using the web based infrastructure which includes features such as housing aggregate and tabulated data provided by students. Examples of this include faculty 
downloading questions, either as a Microsoft Word document or PowerPoint slides, used on a homework set, test, quiz, or in class with an external clicker system. This form of use does not expose students to the site. Even at the basic level of using offline, instructors already using peer instruction or active learning with concept questions need only make minor changes to current practices and the AIChE Concept Warehouse may save them preparation time.

2. Online refers to using the website infrastructure and features. A major benefit of this mode of use is the ability to view results from assignments, which are presented aggregated, tabulated, and archived for later use and are available for download in Microsoft Excel format. If an instructor wants to use more of the features available online, instead of downloading questions they can integrate the use of clickers or have students log in and answer ConcepTests and inventories on their laptops or smart phones (either in-class or for homework). If instructors solicit responses via laptops or smartphones, they can prompt short answer explanations and confidence follow-ups in addition to the multiple choice answers. Such written reflection is perceived by students as helpful. ${ }^{9}$ These more involved features require students to interface with the website.

Table 1. Predicted modes of use of the AIChE Concept Warehouse and their description.

\begin{tabular}{|c|c|c|}
\hline $\begin{array}{l}\text { Mode of } \\
\text { Use }\end{array}$ & & Description \\
\hline Offline & & $\begin{array}{l}\text { Download questions via Microsoft Word or PowerPoint to use on } \\
\text { homework, tests, or with external clicker systems }\end{array}$ \\
\hline \multirow[t]{3}{*}{ Online* } & Homework & Used online outside of class through student interface as homework \\
\hline & $\begin{array}{l}\text { In-Class, with Laptops or } \\
\text { Cell Phones }\end{array}$ & $\begin{array}{l}\text { Used in class allowing short answer explanations and confidence follow- } \\
\text { up }\end{array}$ \\
\hline & $\begin{array}{l}\text { In-Class, with Turning } \\
\text { Point Clickers }\end{array}$ & $\begin{array}{l}\text { Used in class with Turning Point clickers using the AIChE Concept } \\
\text { Warehouse java applet }\end{array}$ \\
\hline
\end{tabular}

* requires students to interface with the site

\section{Diffusion of Innovations}

In this paper we use Diffusion of innovations, a theory put forth by E.M. Rogers in his first book on the topic in 1962. ${ }^{1}$ Diffusion of innovations has been used as a theoretical framework for decades and has accounted for more than 5,000 publications in the field. According to Rogers "diffusion is the process in which an innovation is communicated through certain channels over time among the members of a social system. [p. 5]"1 The four elements that are important in this definition are: (1) the innovation, (2) communication channels, (3) time and (4) social system [p.11]. Characteristics that contribute to the rate at which an innovation is adopted include relative advantage, compatibility, complexity, triability, and observability. The innovationdecision process used by an individual in consideration of adopting an innovation consists of five stages "(1) from first knowledge of an innovation, (2) to forming an attitude toward the innovation, (3) to a decision to adopt or reject, (4) to implementation of the new idea, and to (5) confirmation of this decision. [p. 990]" ${ }^{10}$ Each of these elements have their own characteristics that contribute to the rate at which an innovation is adopted, however we will focus on the social system and the role it plays with diffusion networks. 
A social system is defined as "a set of interrelated units that are engaged in joint problem solving to accomplish a common goal. [p. 23]"1 This system is embedded within a structure which is composed of patterned social relationships and/or interpersonal networks. Within these social systems, it is often the innovator who is seen as an outsider and therefore her/his role within the diffusion process is limited. In order to promote change, individuals who are influential are called opinion leaders. Rogers states that this position is earned and maintained through "technical competence, social accessibility, and conformity to the system's norms [p. 27]" and that these opinion leaders have an important effect on the rate of adoption.

Borrego et al. used Diffusion of innovations as a framework to survey department chairs and investigate faculty awareness and adoption of a wide variety of research-based instructional strategies in engineering education. ${ }^{11}$ They found three major types of factors that contribute to the decision to adopt innovations: ${ }^{11}$

- The most prevalent type of factor was resources (e.g., funding, computers, classroom and laboratory space, etc.).

- Faculty member related issues occurred as the second most common type of factor, and included: time for preparation, management of labor-intensive innovations, culture of the faculty members' environment, "resistance to change, marginalization of teaching in promotion and tenure, and skepticism regarding evidence of improved student learning. [p. 199]"11

- The third type of factor, student-related aspects, included advantages of innovations, such as improved student learning and improved student satisfaction and barriers such as student resistance.

In addition, they emphasize "the importance of disciplinary networks and opinion leaders who are similar to (i.e., practicing in the same discipline as) potential adopters. [p. 200]" ${ }^{, 11}$ In another study, Borrego et al investigated the innovation-decision process in engineering education and highlighted word of mouth, workshops, and literature as the most common diffusion channel to raise awareness. ${ }^{12}$ Other studies in educational contexts have also emphasized the important role opinion leaders play in the promoting adoption of a new innovation. ${ }^{13,14}$ In our previous work, we found that one-on-one, in-person diffusion channels appeared to be more effective than impersonal emails at attracting users that demonstrated a high level of engagement with the AIChE Concept Warehouse. ${ }^{15}$

In this paper we build on our previous work and use Diffusion of innovations to investigate diffusion networks, communication channels, and opinion leaders. The nature of diffusion networks is important to understanding the diffusion process. To understand these networks communication network analysis, which is a method of research to such for the communication structure within a social system, is done. Through network diagrams we are able to identify diffusion networks of users of the $\mathrm{CW}$ and the growth that has occurred over 18 months, as well as the key users who have helped to propagate this innovation.

\section{Methods}

Network analysis was performed including all CW instructor applicants to examine the diffusion networks through which the CW has propagated (research question 1), to examine how users are connected and to identify the opinion leaders that have promoted its propagation (research 
question 2). This study utilized answers to a question on initial AIChE Concept Warehouse applications and general usage data from the CW including date stamps, number of logins, and number of questions downloaded or assigned online. This data was collected and housed in the CW database. Answers to the question "How did you hear about us?" were analyzed using an emergent coding process. Categories representing diffusion channels were identified and network analysis was performed. Network diagrams or sociograms were created for the CW representing different points in time.

A network diagram consists of nodes, or circles in our case, and edges. Each node represents an instructors who applied for, used, or referred an instructor to the CW $(\mathrm{N}>300)$ or an event that appears to have garnered new applicants. These users, instructors that have been given an account, consist primarily of instructors who teach chemical engineering courses, but also include instructors of chemistry, mechanical engineering, physics, and math courses. Nodes in our diagrams have two additional properties: node color represents how a user learned about the CW and node size represents the level of activity of the particular user. If applicants cited multiple events or channels in their answer to "How did you hear about us?" their node was color coded as the event or channel either most prominent (e.g., a response such as, "I heard about it from this year's ASEE conference, but I think I also got emails about it at some point," would be color coded as conference) in the response or, in cases where all channels appear equally prominent, as the event closest in time to the application date. Developers of the CW are represented by nodes towards the center of a diagram. Table 2 summarizes the node color categories. Node size was calculated as an equally-weighted average of three indicators of activity level: number of logins, number of questions downloaded (via Microsoft PowerPoint or Word), and number of questions assigned as online assignments. This weighted average is an approximate indicator of activity and broadly represents the different types of activity common in the different modes of use.

Table 2. Summary of node and edge color categorization.

\begin{tabular}{|l|l|l|}
\hline $\begin{array}{l}\text { Node or } \\
\text { Color }\end{array}$ & Category Name & Category Description \\
\hline Purple & Colleague & $\begin{array}{l}\text { Communication channel was word of mouth via some colleague. The colleague } \\
\text { may have been a developer of the tool, a user of the tool, or in a couple of cases a } \\
\text { non-user. }\end{array}$ \\
\hline Dark Green & Workshop & $\begin{array}{l}\text { Either events that were specified by applicants as workshops or to events that } \\
\text { developers described as workshops. These events typically occur over a } \\
\text { relatively short duration (a few hours to a couple of days). }\end{array}$ \\
\hline Dark Blue & Conference & $\begin{array}{l}\text { Any conference where the CW was presented. Includes annual conferences for } \\
\text { the American Society for Engineering Education and the American Institute of } \\
\text { Chemical Engineers. }\end{array}$ \\
\hline Yellow & Email & $\begin{array}{l}\text { Includes any emails sent out by the developers as promotional material to raise } \\
\text { awareness of the tool such as flyers, newsletters, etc. Also includes when an } \\
\text { applicant specified email, but no other details. }\end{array}$ \\
\hline
\end{tabular}




\begin{tabular}{|l|l|l|}
\hline & Grants & \\
\hline Light Blue & Website & $\begin{array}{l}\text { Any internet originated channel, such as Google or other internet searching, } \\
\text { explicit identification of the CW website, and other websites. }\end{array}$ \\
\hline $\begin{array}{l}\text { Light } \\
\text { Green }\end{array}$ & $\begin{array}{l}\text { Professional } \\
\text { Program }\end{array}$ & $\begin{array}{l}\text { Two types of professional programs were included, both of which featured the } \\
\text { CW as a small part of a multi-week engagement designed to provide instructors } \\
\text { with professional development regarding their teaching practices. }\end{array}$ \\
\hline Brown & Publisher & Textbook publishers. \\
\hline White & Non-user & $\begin{array}{l}\text { An individual that was referenced by an applicant but that has never applied for } \\
\text { or gotten a CW user account. }\end{array}$ \\
\hline Light Gray & Unknown & $\begin{array}{l}\text { No source of information about how the applicant heard about the CW was } \\
\text { stated on the application or noted in any other documentation. }\end{array}$ \\
\hline Black & Developer & $\begin{array}{l}\text { One of the individuals on the initial CW grant that has contributed to CW } \\
\text { development since inception. }\end{array}$ \\
\hline
\end{tabular}

Edges represent the relational links between nodes, and are seen as a line between circles. Each edge has a color that corresponds to the communication channel through which the outermost node of the two being linked learned about the CW. Figure 2 walks through an example diagram. In this example, a developer (illustrated by a black node) told two colleagues about the Concept Warehouse. These two additional users are represented by purple nodes, indicating that they heard about the Concept Warehouse through the "colleague" communication channel. The edges connecting these users to the developer reinforce the "colleague" channel and are also purple. In this example we can also see that the two additional users have different levels of activity with respect to their use of the Concept Warehouse. The larger purple node has a higher level of activity than the smaller purple node.

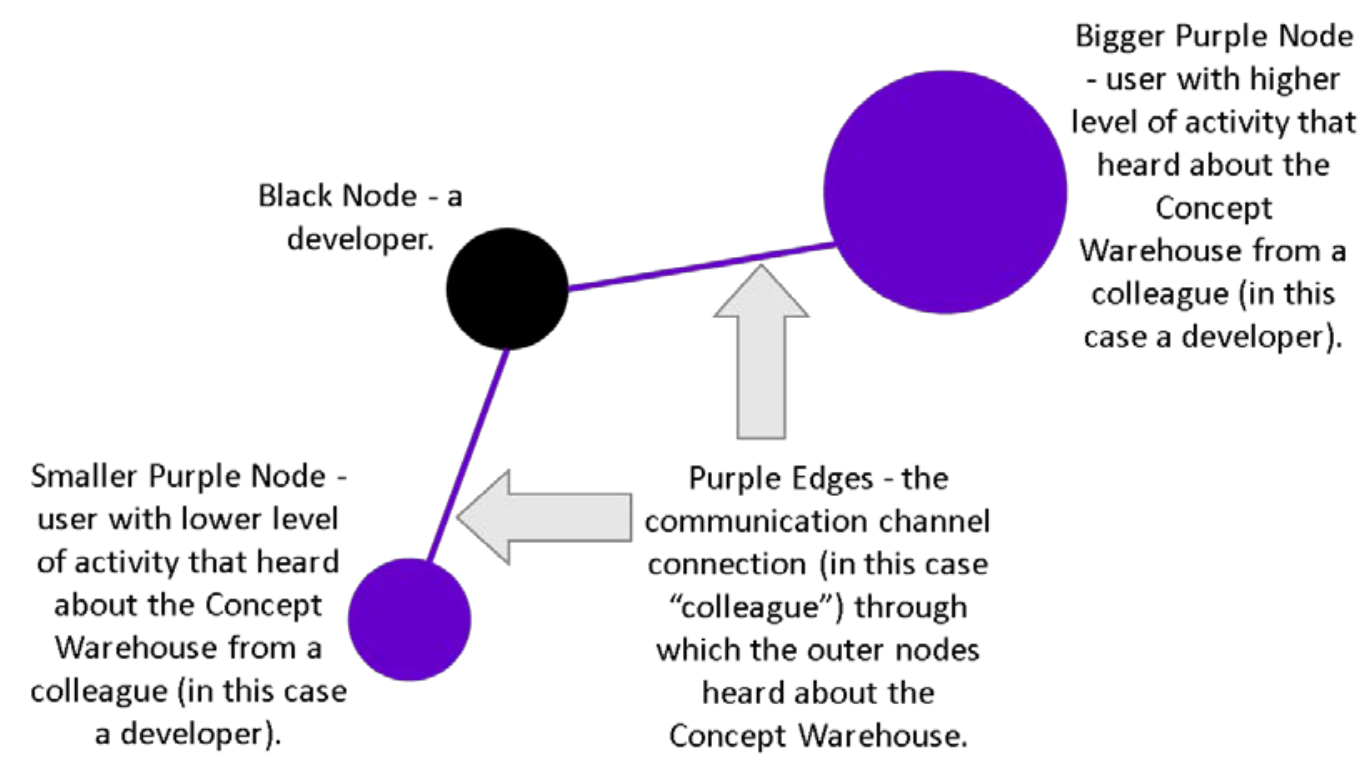

Figure 2. Illustration of how our network diagrams are constructed. 
These diagrams show how each user learned about the tool, what each user's level of activity was at a given point in time, and how they contributed to the spread of the CW. These diagrams allow us to see how the CW has spread over time and through what channels. In addition, the diagrams assist in the identification of opinion leaders by showing how users connect to each other and to communication channels or events.

\section{Results \& Discussion}

Seven network diagrams were constructed that represent different points in time, the first illustrating the CW network at launch and the remainder illustrating the CW network in threemonth intervals until 18 months post-launch. A subset of four diagrams are presented in Figure 3, showing the CW network from launch to 18 months post-launch in six-month intervals. As described in Table 2, each node and edge color signifies a different communication channel with the size of each node corresponding to the activity level of the represented user.

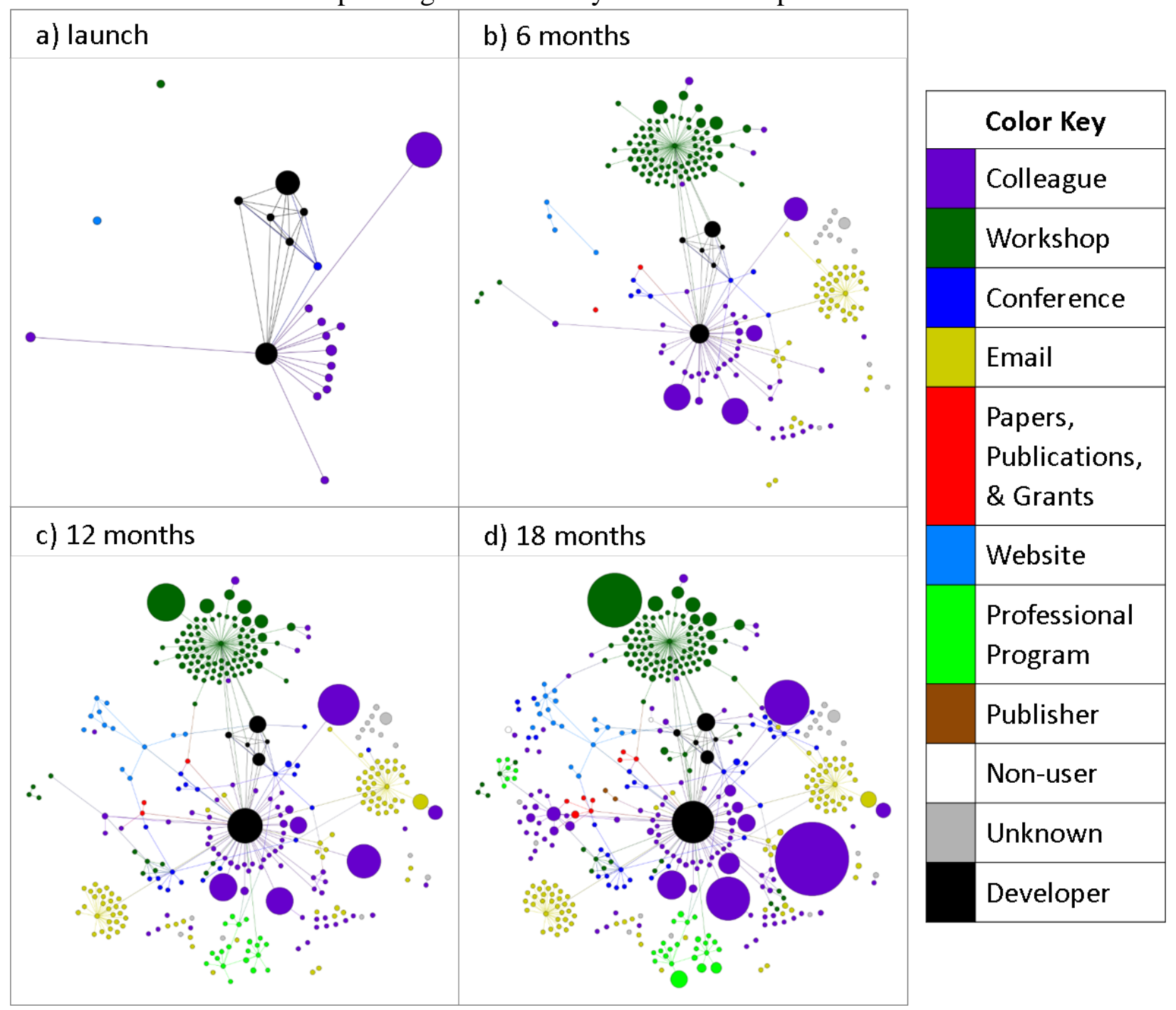

Figure 3. Network diagram tracking the communication channels and user activity for CW accounts at four different points in time. Each dot (or node) represents a user or event and the lines (or edges) represent the communication channels through which users are connected to each other and/or events. Larger dot sizes represent a higher level of Concept Warehouse use. 
Considerable growth can be seen over the initial 18 months of this tool's public availability. In addition, the network diagrams clearly illustrate the flurry of new applicants associated with each propagation activity the developers engage in. For example, the two large clusters of yellow nodes show applicants that signed up as a result of dissemination of two different email list flyers. The majority of high-activity users (the largest node size) learned about the tool through one-on-one interaction via discussion with a colleague or hands-on experience in workshops or longer professional development programs. This result is consistent with earlier findings on the effectiveness of communication channels. ${ }^{15}$

In addition to the substantial growth of the CW observed over its first 18 months of public availability, three additional aspects appear to be changing with time: we begin to see the emergence of potential opinion leaders, we can see the influence of professional development activities (short workshops and longer programs) in the tool's growth, and communication channels cited as propagating the tool begin to be less directly connected to the developers. Each of these aspects is described in more detail below.

\section{Opinion leaders}

We have identified two potential opinion leaders. These individuals were labeled potential opinion leaders because they were referenced by at least four other individuals on applications. The first potential opinion leader is shown as a medium-sized purple node in part (a) of Figure 4. This individual has used the CW for a long period of time and has been noted as a source of information about the CW by other instructors, beginning shortly after the tool's launch. This individual was referenced by eleven applicants as their source of information about the CW. In addition, this individual has incorporated the $\mathrm{CW}$ as part of multiple faculty development activities. 


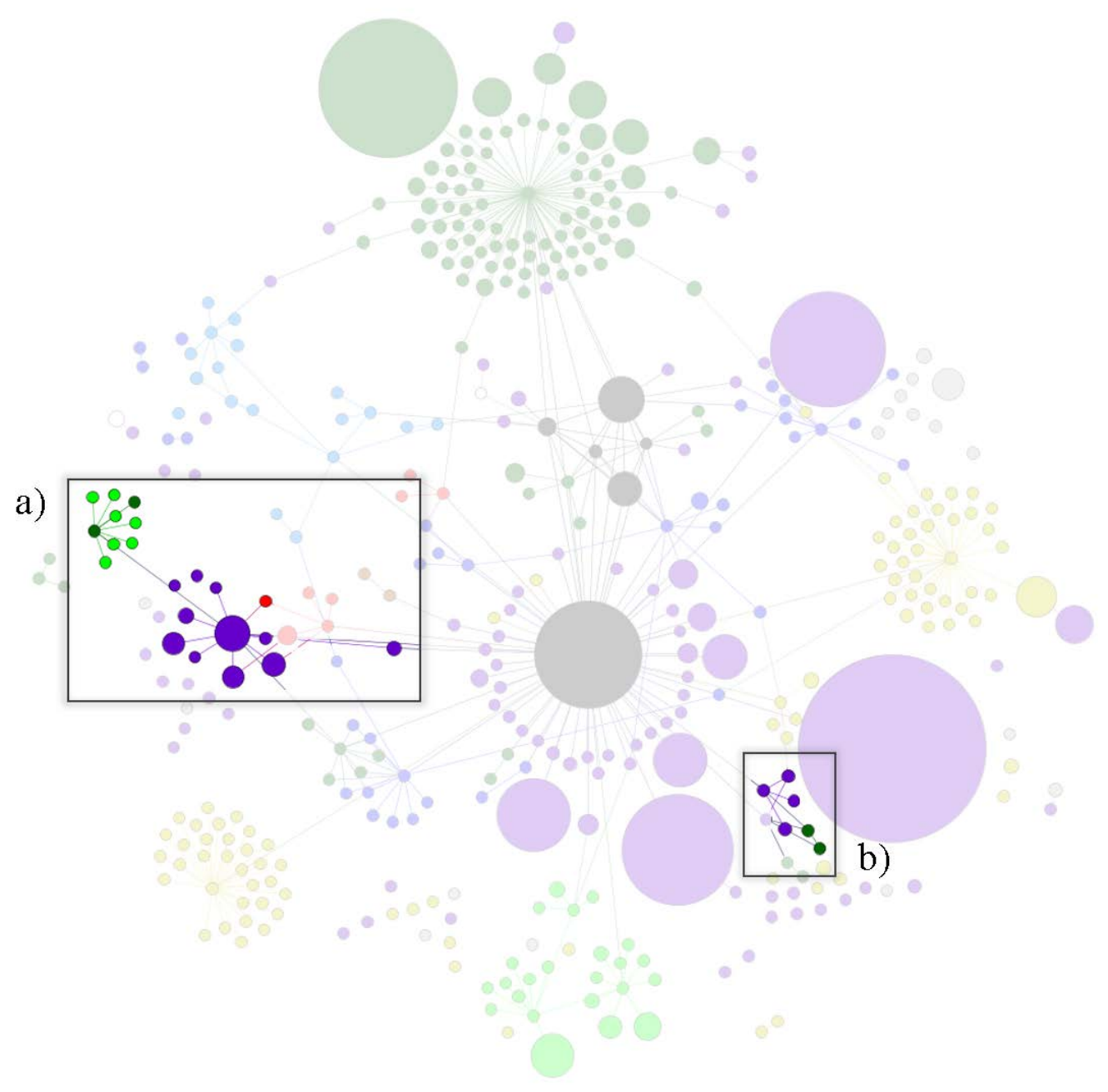

Figure 4. Identification of potential opinion leaders

The second potential opinion leader, while not nearly as active as the first, has been cited as a source of information for four individuals and has also apparently discussed the CW while hosting a professional development activity. We intend to monitor the network diagrams as the tool continues to grow in order to identify additional potential opinion leaders. In addition, we plan to invite the two identified potential opinion leaders noted here to be interviewed. We hope to gather more information about their propagation activities so that we can better support them in their efforts.

\section{Participants in professional development activities}

Along with individual opinion leaders that appear to be influencing the network, professional development activities have also had a substantial influence. Figure 3d presents two of these activities with a workshop represented as a cluster of dark green nodes at the top of the diagram and a longer program represented as a cluster of light green nodes at the bottom of the diagram. In Figure 5 we focus on the evolution of the light green cluster because of the apparent quick adoption indicated by the three growing nodes at the bottom of the figure. In only three to six months after learning about the tool, three of these professional development program 
participants appear to have implemented it. While we cannot be sure, perhaps the long-term involvement in a pedagogically-focused, professional development program provided these individuals with the support necessary to feel comfortable and confident in using this tool so quickly. These individuals, like the potential opinion leaders identified above, are excellent candidates for a follow-up interview study. We may be able to learn more about supporting such seemingly quick adoption from these individuals' experiences. In addition, many of the participants in that professional development activity appear to have a low-level of activity. A subset of these individuals may also be excellent candidates for interviews as they may be able to provide insight into the barriers they faced and reasons they chose not to implement the tool.

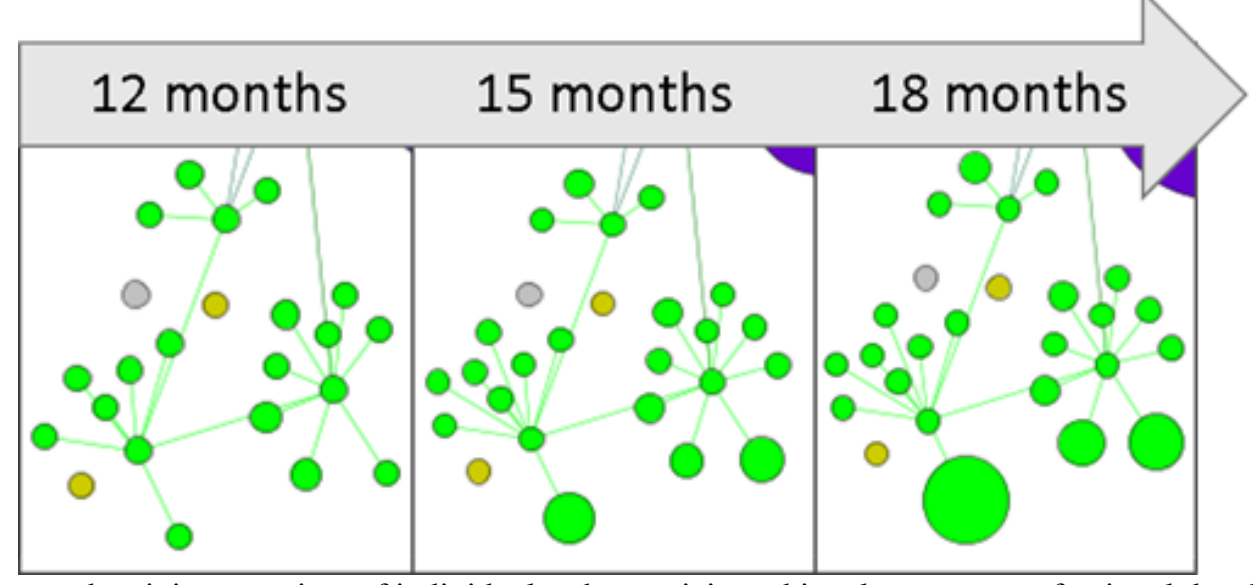

Figure 5. Increased activity over time of individuals who participated in a long-term professional development program.

Applicants informed not through developer channels

One somewhat surprising result of these network diagrams was the apparent emergence of communication channels where the developers invested little or no continuous, visible activity. Four of these communication channels were identified. They are shown in Figure 6 and discussed in more detail in the subsections below.

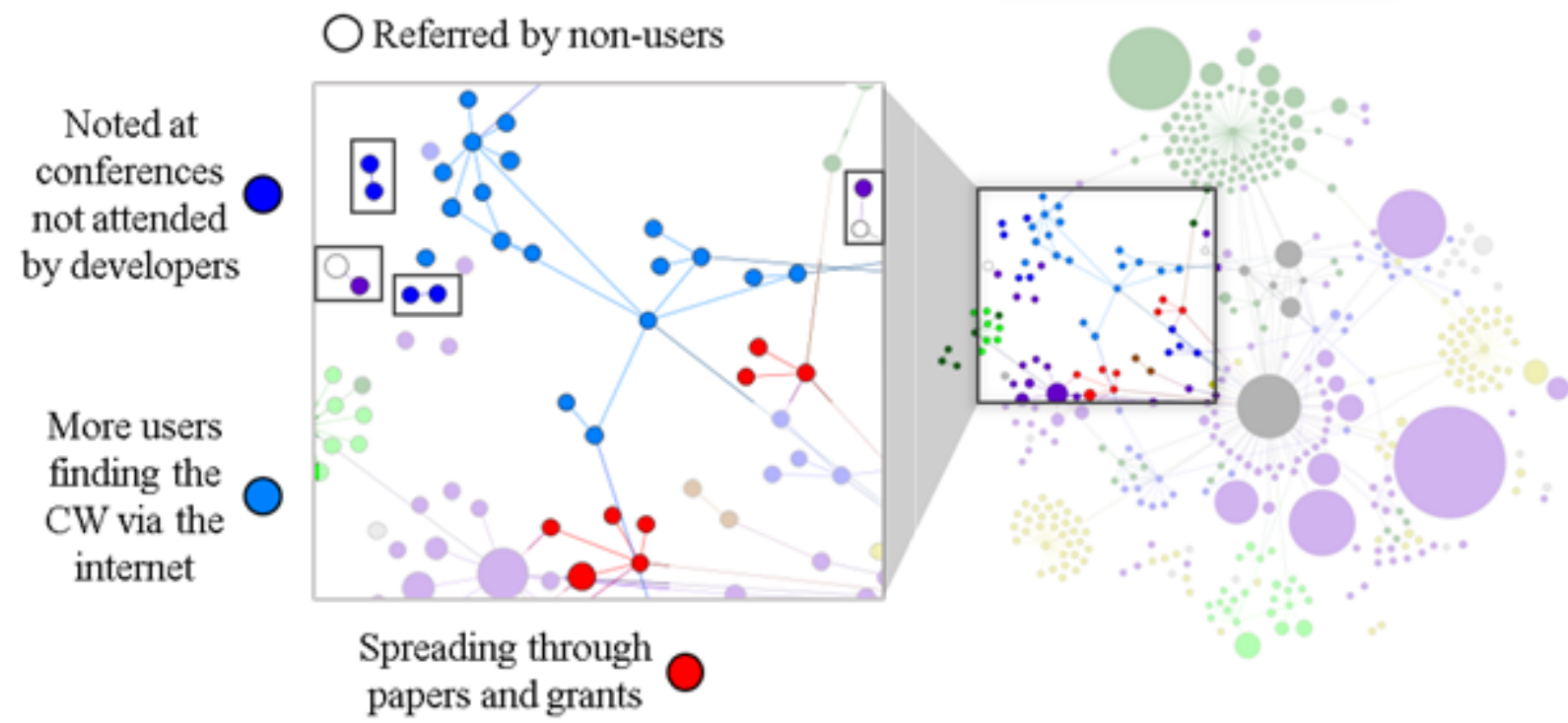

Figure 6. Four communication channels were identified that involve little or no continuous active involvement by the developers. 
Referred to instructors by non-users - Two non-users (shown as white nodes with a black border) were referenced as colleagues that informed an applicant about the $\mathrm{CW}$. It is possible that the applicants remembered their referrers incorrectly. However, it is also possible that awareness of the tool is becoming more widespread such that even users that don't see a direct application of the tool for themselves recognize it as potentially valuable for colleagues.

Noted at conferences not attended by the developers - Two conferences (both the conference and the applicant are shown as dark blue nodes) were referenced by recent applicants (nodes only present in the most recent network diagram) that the developers had not attended. These two are the first instances of a conference being cited where no developer presented work on the tool or hosted any form of workshop or panel session. This type of activity may also be an indicator that the $\mathrm{CW}$ is reaching more widespread visibility.

An increasing number of users is finding the CW via internet sources (shown as light blue nodes and edges) - A somewhat less surprising observation is the increase in reference to internet searching and websites in general for their role in connecting instructors to the CW. As the tool becomes more commonly known, is present in more publications, and accessed by more users, it should become easier to find with search engine keyword searches. We expect this apparent trend to continue.

Spreading through papers and grants - While papers and grant proposals and reports require effort during preparation, once they are submitted and accepted, the authors are required to provide very little, if any, continuing support. However, these documents are archived and continue to be available via internet searching, archival journals, and grant proposal and report databases. Similar to the internet searching, if there are more materials readily available, a connection is more likely to be made. This result suggests that publications can raise awareness regarding a tool. While internet searchability and publications may serve a purpose in raising awareness, neither communication channel has yet to result in attracting users with a high level of activity.

\section{Conclusions \& Implications}

Over the first 18 months of the public availability of the AIChE Concept Warehouse there has been a substantial increase in the number of users. In this paper we have shown how the user network has changed over time and which communication channels have been referenced as a source of information and referral for the tool. Nine categories of communication channels were noted including: email, internet searching, reading papers and grants, talking with a publisher, word-of-mouth via colleagues, and attending workshops, conferences, or longer-term professional programs. The current study reinforces findings of a previous study suggesting that, at least at this stage of the tool's life, one-on-one interaction appears to be more effective at promoting high levels of tool use activity. We have also identified two potential opinion leaders who have both suggested the tool to colleagues and incorporated it into professional development activities that they hosted or took part in leading. Finally, we present a somewhat surprising result that applicants are citing as their point of referral communication channels with which the developers have little or no continuous active involvement. This result may be a sign of overall increased awareness of the tool. It is probably too early in the tool's life to know. We 
have presented a few snapshots of the early stages of an educational innovation's life. These results and the methods used in this paper can serve as a starting point to provide ideas for other innovators embarking on the launch of a new educational innovation or considering examining the early stages of propagation of a tool. However, the results presented prompt a need for further study to more fully understand the system within which the AIChE Concept Warehouse is propagating and the characteristics and motivations of its users.

\section{Future Work}

The network diagrams presented a very useful tool for quickly visualizing the propagation of the AIChE Concept Warehouse. They have illuminated several points of interest that warrant further investigation. For example, communication with the potential opinion leaders identified in this study may be able to provide insight into other propagation strategies and ways to support their efforts and minimize barriers. In addition, the users in the professional development program that so quickly learned about and implemented the tool may be able to offer recommendations for how we can further support such quick implementation. Users that appear to be static should be sampled to identify barriers to adoption and implementation.

\section{Acknowledgments}

This material is based upon work supported by the National Science Foundation under Grant No. DUE 1023099, 1022957, 1022875, 1022785. We also acknowledge a supplement to grant DUE 1023099 to participate in the I-Corps for Learning program. Any opinions, findings, and conclusions or recommendations expressed in this material are those of the authors and do not necessarily reflect the views of the National Science Foundation.

We would also like to acknowledge all of the individuals who are using the AIChE Concept Warehouse and those who have provided feedback to improve this tool, particularly those who participated in this study. We would also like to acknowledge the people who supported this work with their time and help.

\section{References}

1. E. M. Rogers, Diffusion of innovations, Free Press, New York, 2003.

2. E. National Academy of, Educating the engineer of 2020 : adapting engineering education to the new century, National Academies Press, Washington, DC, 2005.

3. National Research Council. How People Learn: Brain, Mind, Experience, and School: Expanded Edition. Washington, DC: The National Academies Press, 2000.

4. D. Hestenes, M. Wells and G. Swackhamer, The Physics Teacher, 1992, 141-158.

5. A. Elby, American Journal of Physics, 1999, S52.

6. R. M. Felder and R. Brent, Journal of Engineering Education, 2005, 57-72.

7. C. Crouch, J. Watkins, A. Fagen and E. Mazur, Research-Based Reform of University Physics, 2007.

8. A. Fagen, C. Crouch and E. Mazur, The Physics Teacher, 2002, 206-209.

9. M. D. Koretsky and B. J. Brooks, Chemical Engineering Education, 2012, 46, 289-297.

10. E. M. Rogers, Addictive behaviors, 2002, 27, 989-993.

11. M. Borrego, J. E. Froyd and T. Simin Hall, Journal of Engineering Education, 2010, 99, 185.

12. M. Borrego, S. Cutler, J. Froyd, M. Prince and C. Henderson, in Australasian Association for Engineering Education Conference 2011: Developing engineers for social justice: Community involvement, ethics \& sustainability 5-7 December 2011, Fremantle, Western Australia, Engineers Australia, 2011, p. 448. 
13. M. Hall \& K. M. Elliott, Journal of Education for Business, 2003, 78:6, 301-307, DOI: $10.1080 / 08832320309598617$

14. M.M. Jennings \& D. J. Dirksen. Facilitating change: A process for adoption of web-based instruction. p. 111-116 in Web-based instruction Educational Technology Publications, Inc, Englewood Cliffs, New Jersey,1997.

15. D. Gilbuena, C. Smith, B. Brooks, M. Miletic, \& M. Koretsky, Research in Engineering Education Symposium, Kuala Lumpur, Malaysia, 2013. 